\title{
An experimental validation of residual stresses in weld clad pipelines
}

\author{
G. Schnier, J. Wood \& A. Galloway \\ JW Analysis, UK
}

\begin{abstract}
The nature and distribution of residual stresses are invariably critical for fatigue life with dissimilar material joints often inducing high tensile residual stresses. A fatigue-resistant concept of weld cladding process pipelines, producing compressive residual stresses, is under investigation to examine how these stresses may be influenced. Simplified weld cladding simulations have successfully illustrated the development and distribution of residual stresses through the joint. The study has highlighted the importance of accurate material data for clad and substrate materials with current analysis assumptions in a simple thick-walled pipe discussed. Experimental validation, using ICHD, measured residual stresses with depth on weld clad specimens, resulting in good correlation between simulation and experiment for a nickel-chromium-based superalloy clad on low alloy carbon steel as discussed. Future work, including a full 3D representation of the cladding process and a comparison of residual stress measurement methods, are also discussed.
\end{abstract}

\section{INTRODUCTION}

Residual stresses are typically described as the stresses in a body post-manufacture with no external loading or constraints present. Depending on the nature of these residual stresses, they can be beneficial or detrimental to the fatigue life of a component. It has been found that many machining processes induce tensile residual stresses which in turn shorten fatigue life. Tensile residual stresses also arise in dissimilar joints and can be as high as yield for the materials in question. From a fatigue perspective, it is desirable to maintain compressive residual stresses to increase fatigue strength, particularly at the surface of a component where operational stresses are often at their highest.

Residual stresses are elastic and self-equilibrating in nature and arise from various mechanisms in the case of dissimilar weld cladding: a surface "heattreatment effect" due to materials varying with temperature affecting cooling rates and constraint levels, a "pre-heat shrink-fit effect" accounting for effective shrinking of the cladding relative to the substrate affected by material and temperature dissimilarity and "spatial and temporal variation" of the clad deposition process and subsequent cooling causing changes in microstructure through phase transformation and chemical reactions.

This paper presents results from initial simplified 2D models validated with experimental residual stress measurements and discusses the progression to a full 3D model. As will be shown, pipeline weld cladding and dissimilar material joints in general will also display significant discontinuity stresses at the interface between the dissimilar materials. Fatigue cracking may therefore occur sub-surface.

\section{DISSIMILAR JOINT SIMULATION}

The weld clad pipeline model currently takes the form of a thermal transient stress analysis of a twodimensional axisymmetric finite element model (Fig. 1). A simplified process of an entire clad layer being deposited onto the outer diameter of a preheated thick-walled pipe at melt temperature at time $t=0$ seconds has been employed as an initial study. This modelling technique assumes that there is no variation in temperature or stress field in the axial and hoop directions as a function of time. The entire component was then subjected to slow cooling on the inner and outer surfaces using a convective heat transfer coefficient $h=10 \mathrm{~W} / \mathrm{m}^{2} \mathrm{~K}$ and a bulk temperature of $25^{\circ} \mathrm{C}$, with latent heat effects being neglected. The $C A X 4 T$ Abaqus element type was utilised with bi-linear displacement and temperature shape functions.

The radial edges of the model were insulated to simulate no axial heat transfer and plane strain conditions applied on the radial lines (Fig. 2). 


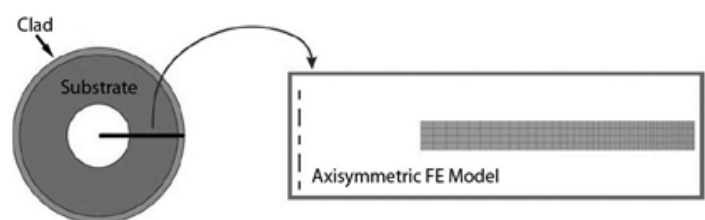

Figure 1. Axisymmetric FE Model

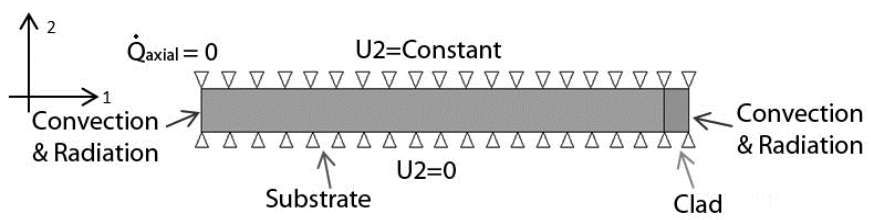

Figure 2. Axisymmetric Model Boundary Conditions

Although this model is a simplification of a process with spatially and temporally varying deposition, it is representative of methods such as evaporative deposition or spray coating followed by sintering. In the following sections it will be shown however that it also provides good correlation to experimental measurements for the spatially and temporally varying weld deposition process used.

An elastic-perfectly plastic material model was assumed with temperature dependent thermal and mechanical material properties. Focus was placed on coefficient of thermal expansion, specific heat, thermal conductivity, Young's modulus and yield stress. These properties primarily govern the modelling of the thermal event, degree of constraint and therefore the development of residual stresses (Ahmed et al. 2007), with further contributing factors discussed in the reference given. Poisson's ratio and emissivity were assumed to remain constant. The nature of residual stresses differs depending on the state of a component, as discussed by Withers and Bhadeshia, with misfit resulting from different parts, phases and regions highlighted as the key characteristic influencing residual stresses (Withers $\&$ Bhadeshia 2001). Phase transformations in steels have been shown to influence residual stress development in X70 pipeline steel (Onsoien et al. 2010) for example.

Figure 3 shows a large discontinuity in the stress distribution in the vicinity of the low alloy carbon steel substrate and clad interface for both pre-heat and material combinations, with a change from compressive to tensile stresses. At the idealised interface between the substrate and clad there is a step change in the stresses with the nickel-chromiumbased superalloy clad showing a reduction in residual stress, which remains significantly tensile throughout the clad. The form of residual stress graph for the nickel-chromium-based superalloy clad is indicative of yielding and reflects the elasticperfectly plastic model used.
The stainless steel clad simulation results on the other hand show a further increase in residual stress in the clad, which is unexpected and is contrary to the experimental results obtained, which show a similar value of residual stress throughout the clad but compressive in nature.

\section{EXPERIMENTAL VALIDATION}

\subsection{Background}

Incremental centre hole drilling (ICHD) is a relaxation technique used for residual stress measurement through the measurement of local displacement in the vicinity of a drilled hole (ASTM International 2008). Material removal through the drilling of a hole into a component allows the consequent movement of remaining material to be measured and residual stresses to be deduced. This occurs most commonly through the application of a strain gauge to obtain strain values throughout the material removal process, which can be converted into stress values. Through the drilling of the hole, additional stresses can be induced, however the application of high-speed air-driven turbines operating at speeds up to $400,000 \mathrm{rpm}$ (Grant et al. 2006) minimises this effect.

\subsection{Hole-drilling method}

Specialised three element strain gauge rosettes are applied to the surface after appropriate surface preparation techniques. The method of surface preparation is dependent on the initial roughness of the surface and could require machining prior to finishing with fine grain sandpaper. Wiring and balancing of the gauges follows the laying of the gauge after which the fixture can be applied to the component by means of cementing the detachable legs prior to assembling the drilling kit.

Strain readings are then recorded at incremental depths, with accuracy of the readings decreasing with increasing depth due to the travelling further from the applied strain gauge on the surface. The maximum depth to which strain readings can be used for the purpose of residual stress measurements is to one half of the diameter of the drilled hole (Grant et al. 2006).

Residual stress measurements were obtained for both cylindrical and rectangular specimens. On the cylindrical specimens, gauges were installed at the centre of the outer diameter of the component at two diametrically opposed circumferential locations to demonstrate variability in cladding and measurement process. For the rectangular specimens, gauges were applied along the centre-line to allow effects of global bending to be deduced. 


\subsection{Strain measurement conversion}

Three main conversion methods exist for the conversion of strain measurements, namely the Equivalent Uniform Stress (EUS), Power Series and Integral methods (Schajer 1988). Schajer notes that the EUS and Power Series are approximations of the Integral method, which provides the most acceptable interpretation of uniformly varying residual stresses through applying the least smoothing of results and is therefore applied as the standard calculation procedure for residual stresses using ICHD.

\section{RESULTS}

Figure 4 shows good agreement between the nickelchromium-based superalloy cladding simulation results and experimental measurements using ICHD. The two sets of experimental results are for two different diametrically opposed positions on the centre of the cylindrical specimen. The difference in the experimental results is indicative of the effect of process variability. The reduction in both sets of experimental results over the first 200 microns will largely be due to the machining of the weld profile to provide a flat surface for strain gauging.

\subsection{Comparison of surface preparation techniques}

Three surface preparation techniques were used in preparation for applying the strain gauge. The first was the use of a hand-held compressor driven stone grinder followed by fine finishing using P400 fine sand paper. The second technique was $\mathrm{CNC}$ milling at 500rpm using $10 \mu \mathrm{m}$ increments to a depth of $0.5 \mathrm{~mm}$ for the nickel-chromium-based superalloy clad and $0.7 \mathrm{~mm}$ for the stainless steel due to varying clad profiles. Lastly surface preparation using a PCcontrolled machine tool beginning at $16 \mu \mathrm{m}$ per orbit, decreasing to $8 \mu \mathrm{m}$ and finally $2 \mu \mathrm{m}$ for the final two orbits was applied to minimise machining effects.

From the results shown for a nickel-chromiumbased superalloy clad pipe, it can be seen that in comparing hoop stress values for the three surface preparation techniques the hand grinding technique gives a highly inaccurate representation (Fig. 5). Results for the milling and PC-controlled surface preparation techniques produce a greater degree of correlation as would be expected, with discrepancies arising especially close to the surface however.

Focus is placed on hoop stress values in the following discussion as this stress component presents the greatest contribution to the final stress state.

The results shown for the stainless steel clad pipe (Fig. 6) show a greater variation in the CNC surface preparation method with alarming gradients shown in one milling measurement. This is most probably due to user technique, however the requirement for careful surface preparation is clear.

Overall, the residual stress values for the stainless steel clad material provide a tighter band of results with greater uniformity of stresses over the $1 \mathrm{~mm}$ depth shown. From these results, it can be concluded that there are greater effects due to process variability in the case of nickel-chromium-based superalloy clad.

\subsection{Errors associated with ICHD}

As mentioned in the previous sections, errors arising through the ICHD method can heavily influence strain readings and hence residual stress values. Strain measurement errors can also arise due to user technique, instrumentation errors and additional strains induced through material removal, while hole measurement errors in both the depth and diameter, tapering of the hole and any roundness in the bottom of the hole can influence calculated stress values. Differences can also arise from using different strain gauge types which was the case in the last surface preparation technique of milling depth varying with orbit depth.

Furthermore, errors can arise from deviations in hole roundness and eccentricity errors in the location of the hole with respect to the strain gauge along with material constant errors arising from Young's modulus and Poisson's ratio values applied in the strain-to-stress conversion process (Schajer \& Altus 1996).

\subsection{Additional considerations}

An area that requires careful consideration with regards to residual stresses is that of machining. Any machining of the component after cladding will modify the self-equilibrating residual stress distribution in a thin surface layer and for this reason care must be taken to ensure that any beneficial compressive residual stresses effects are not removed by such an operation. Machining can induce tensile residual stresses, which may also increase stresscorrosion cracking susceptibility in some materials, while also decreasing fatigue life. This was emphasised in a study in 1969 (Paxton \& Procter 1969), highlighting the need for careful consideration of machining processes, through experimental procedures, to ensure an acceptable residual stress state while maintaining adequate corrosion resistance. Investigation into electro-polishing is underway to determine whether this technique, minimising stress state adjustment, can provide further increased accuracy.

Another aspect which could alter surface residual stresses is that of the thermal environment present at the surface during cooling. 
Optimal depth increments have been discussed for differing strain gauge rosette radii and hole radii (Stefanescu et al. 2006). Arbitrary values were applied in this study due to the lack of measuring such precise increments, however in future it would be recommended that a method of determining and measuring such incremental values is employed with reference to the work noted above.

\section{DISCUSSION ON INFLUENCING RESIDUAL STRESSES}

\subsection{Pre-heat temperature}

The effect of pre-heating the substrate to a specified uniform temperature before commencing with the joining process was investigated using two temperature values. The purpose of pre-heating the substrate serves as a method of reducing the heat sink effect and a higher pre-heat would be expected to reduce residual stress levels. A higher preheat however will also increase the pre-heat shrink-fit effect referred to in the introductory section of this paper.

Results show that a lower pre-heat temperature for stainless steel clad specimens results in a higher residual stress level, desirable both economically and for fatigue purposes, however in the case of the nickel-chromium-based superalloy a lower residual stress occurred with a lower pre-heat temperature.

\subsection{Substrate geometry}

The investigation of substrate geometry effects suggests that there are no great variations in residual stresses in the clad pipe and rectangular specimen. Results for low alloy carbon steel clad with stainless steel on the cylindrical and rectangular specimens at a pre-heat temperature of $300^{\circ} \mathrm{C}$ in particular demonstrated that equal residual stress levels are attainable in both geometries providing the thermal transient event is maintained for the differing substrate geometry.

\subsection{Post-weld stress relief}

Post-weld stress relief was unsuccessful for the nickel-chromium-based superalloy clad case, thought to be due to the large mismatch in material properties between these materials. Results for stainless steel clad demonstrate a significant decrease in residual stress values post-stress relief, as well as an almost equi-biaxial stress field further beneficial to fatigue life. The stainless steel and low alloy carbon steel are both high-strength materials with similar metallurgical structure and material property values for CTE and yield stress. However, clearly in the case of stainless steel clad stress relief is not desira- ble as it would lower the high level of beneficial compressive residual stresses.

\section{CONCLUSIONS}

A simplified 2D simulation model of cylindrical dissimilar weld clad process pipeline specimens has shown good agreement with experimental residual stress measurements for nickel-chromium-based superalloy clad on low alloy carbon steel. The lack of agreement for a stainless steel clad has prompted an experimental programme to determine more accurate temperature dependent mechanical property data to increase residual stress representation in the FE model. Published data availability, even for wrought material, in this case was very limited neglecting the phase change present in stainless steel clad and substrate materials assuming instead a linear extrapolation beyond $400^{\circ} \mathrm{C}$.

Simulation models using a step change in material properties at an idealised interface employ a significant approximation to reality. Studies (not reported herein) reveal a variation in elemental composition and grain structure across such junctions, reflected in micro- and nano-hardness measurements. It is likely therefore that other properties used in such simulations will also show a transition (albeit over a relatively short distance). This will have the effect of reducing discontinuity stresses and eliminating any theoretical singularities that may be picked up with very fine finite element meshes.

Experimental residual stress measurements concluded that surface preparation techniques are critical to ensure that the residual stress state in a component is not further altered prior to measurement, therefore rendering harsh hand grinding unacceptable. Additional sources of error were discussed and the sensitivity of readings with user technique highlighted.

Lastly a discussion on influencing residual stresses revealed that a lower pre-heat temperature was more beneficial in the stainless steel and low alloy carbon steel combination, however this was not found to be the case for nickel-chromium-based superalloy and low alloy carbon steel. Substrate geometry effects were found to be negligible and so residual stresses should be attainable in both the cylindrical and rectangular specimens at similar values, while stress relief was unsuccessful in the case of nickel-chromium-based superalloy cladding, although achievable with stainless steel clad material. Experimental variability in the cladding process revealed a much greater effect in the case of nickelchromium-based superalloy cladding in diametrical measurements and along the centre line of the clad plate. 


\section{FURTHER WORK}

Although acceptable representations of the residual stress states in the dissimilar joint were obtained for the nickel-chromium-based superalloy clad, it is important that the model is developed to include a more accurate representation of all relevant temperature dependent material properties. This will provide a better representation of the measured results for a stainless steel clad. In addition, although a simple axisymmetric idealisation has shown good agreement with experiment for the nickel-chromiumbased superalloy clad, a 3D model taking into account both spatial and temporal process variation is underway. Sequential deposition of the clad material is currently being investigated in the $2 \mathrm{D}$ model and this feature of the process will also be included in the $3 \mathrm{D}$ simulation.

It is recognised that it is important in such simulations to capture the thermal behaviour of the joining process (Smith et al. 2011) and so calibration of the 3D simulation will be carried out using extensive temperature information obtained during the cladding process, which also reflects the weld inter-pass temperature used.

Further work is also underway to examine the use of functionally graded material properties in dissimilar joints in an effort to reduce the level of subsurface discontinuity stresses whilst retaining the benefit of any compressive residual stresses that may result in the clad layer. The metallurgy of the joint will also be further examined with emphasis placed on the influence of weld parameters on joint metallurgy.

Due to limitations of ICHD it would be desirable to develop finite element models calibrating the residual stress state further into the component. Alternatively the deep-hole drilling method could be investigated providing residual stress measurements to depths of up to $750 \mathrm{~mm}$ (VEQTER 2013). Further investigation of surface preparation techniques will allow the curvature of cylindrical specimens to be accounted for such that gauges will be laid on a rounded surface as opposed to the milled surface being completely flat.

\section{REFERENCES}

Ahmed, R. et al., 2007. Residual Strain Measurements in Thermal Spray Cermet Coatings via Neutron Diffraction. Journal of Tribology, 129(2), p.411.

ASTM International, 2008. Standard Test Method for Determining Residual Stresses by the Hole-Drilling Strain-Gage Method. ASTM E837-08e1.

Grant, P. V, Lord, J.D. \& Whitehead, P S, 2006. Measurement Good Practice Guide No . 53 - Issue 2 The Measurement of Residual Stresses by the Incremental Hole Drilling Technique,

Onsoien, M.I., M'Hamdi, M. \& Akselsen, O.M., 2010. Residual Stresses in Weld Thermal Cycle Simulated Specimens of X70 Pipeline Steel. Welding Journal, 89, p.127-s - 132-s.

Paxton, H.W. \& Procter, R.P.M., 1969. The Effects of Machining and Grinding on the Stress-Corrosion Cracking Susceptibility of Metals and Alloys. American Society of Tool and Manufacturing Engineers, EM68520.

Schajer, G. S., 1988. Measurement of Non-Uniform Residual Stresses Using the Hole- Drilling Method . Part I Stress Calculation Procedures. Journal of Engineering Materials and Technology, 110(October), pp.338-343.

Schajer, G. S. \& Altus, E., 1996. Stress Calculation Error Analysis for Incremental Hole-Drilling Residual Stress Measurements. Journal of Engineering Materials and Technology, 118(1), pp.120-126.

Smith, M.C., Smith, A.C. \& Wimpory, R., 2011. Review of the NeT Task Group 1 Single Weld Bead on Plate Benchmark Round Robin,

Stefanescu, D. et al., 2006. Improvements in Residual Stress Measurement by the Incremental Centre Hole Drilling Technique. Experimental Mechanics, 46(4), pp.417-427.

VEQTER, 2013. Deep-Hole Drilling. VEQTER Ltd. Available at: http://www.veqter.co.uk/residual-stressmeasurement/deep-hole-drilling [Accessed February 11, 2013].

Withers, P. \& Bhadeshia, H.K.D.H., 2001. Residual stress: Part 2 - Nature and origins. Materials Science and Technology, 17, pp.366-375. 


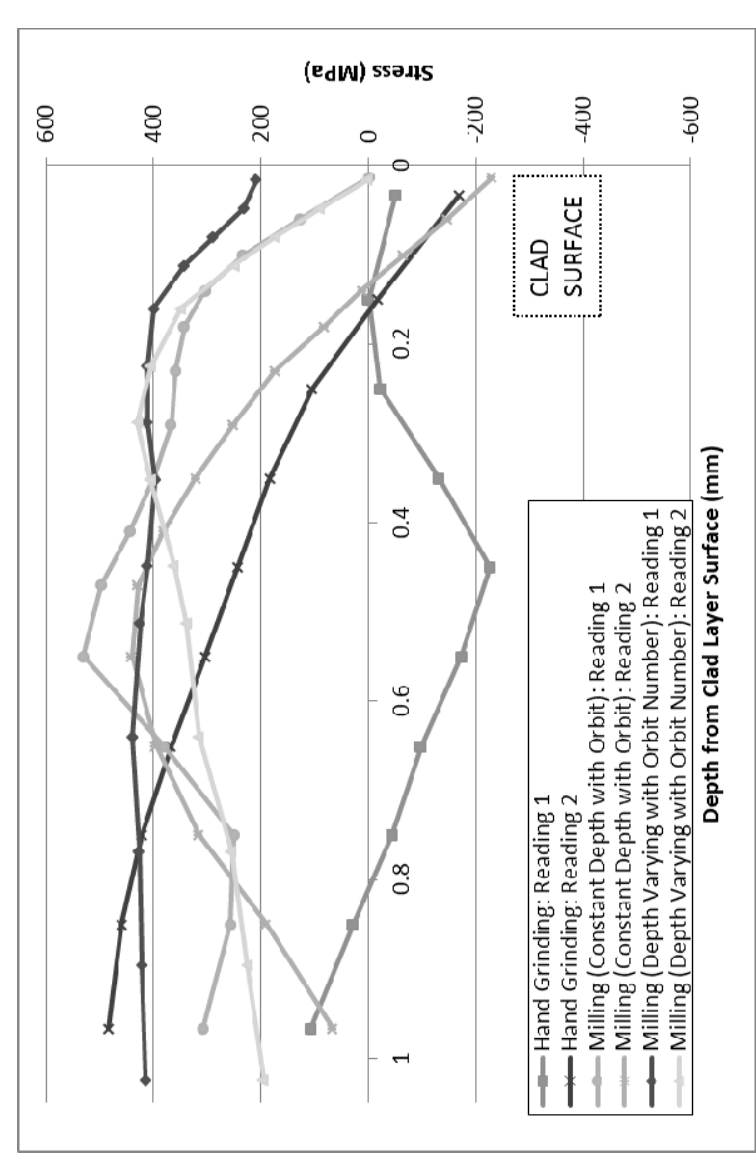

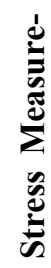
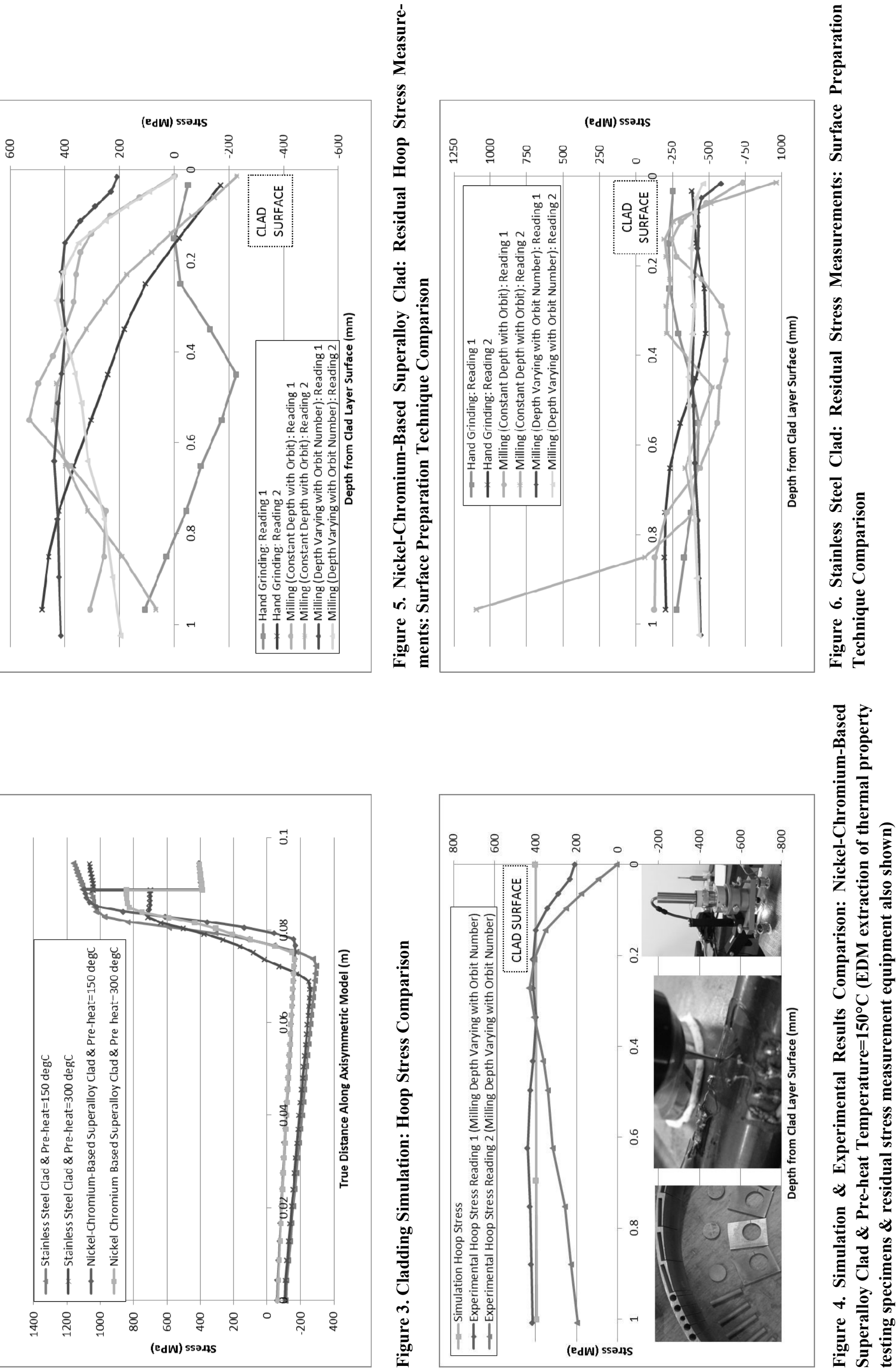